\title{
Note on Legendre's and Bertrand's Proofs of the Parallel-Postulate by Infinite Areas.
}

\author{
By Dr D. M. Y. Sommerville.
}

(Read and Received 8th December 1911).

One of the most plausible of the host of "proofs" that have ever been offered for Euclid's parallel-postulate is that known as Bertrand's, * which is based upon a consideration of infinite areas. The area of the whole plane being regarded as an infinity of the second order, the area of a strip of plane surface bounded by a linear segment $\mathrm{AB}$ and the rays $\mathrm{AA}^{\prime}, \mathrm{BB}$ perpendicular to $\mathrm{AB}$ is an infinity of the first order, since a single infinity of such strips is required to cover the plane. On the other hand, the area contained between two intersecting straight lines is an infinity of the same order as the plana, since the plane can be covered by a finite number of such sectors. Hence if $A P$ is drawn making any angle, however small, with $\mathrm{AA}^{\prime}$, the area $\mathrm{A}^{\prime} \mathrm{AP}$, an infinity of the second order, cannot be contained within the area $A^{\prime} A B B^{\prime}$, an infinity of the first order, and therefore $\mathrm{AP}$ must cut $\mathrm{BB}^{\prime}$. And this is just Euclid's postulate.

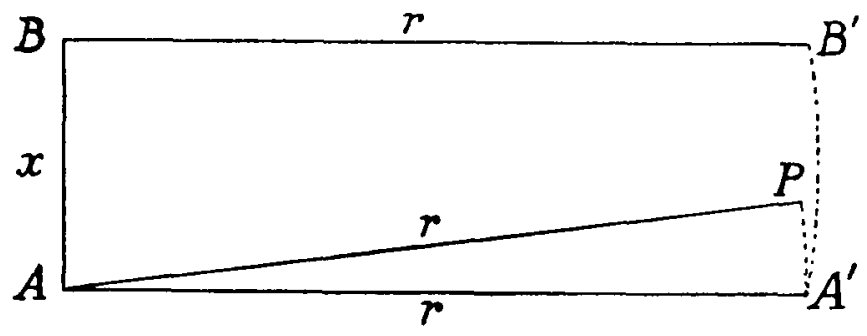

Fig. 1.

- L. Bertrand, Développement nouveau de la partie élémentaire des mathématiques. Genève, 1778., t. II., p. 19. 
The fallacy consists in dealing with infinities as if they were magnitudes of a definite size, whereas the whole question is one of limits.

Without leaving the field of Euclidean geometry it is possible to show that the order of infinity of the area included between two rays proceeding from a point is a variable thing depending upon the way in which the area grows. Thus, taking a line NY and a point $O$, divide $N Y$ into an infinite number of equal parts at $\mathrm{A}, \mathrm{B}, \mathrm{C}, \ldots$, and joint these points to $\mathrm{O}$. The triangles NOA, $A O B, B O C, . .$, are all equal in area. Draw $N^{\prime} \mathbf{Y}^{\prime}$ parallel to $N Y$ and produce the rays from $O$ to cut this line in $N^{\prime}, A^{\prime}, B^{\prime}, C^{\prime}, \ldots$. Then the same thing holds for the triangles $\mathrm{N}^{\prime} \mathrm{OA}^{\prime}, \mathrm{A}^{\prime} O \mathrm{~B}^{\prime}, \ldots$. Let $N^{\prime} Y^{\prime}$ move off to infinity, always remaining parallel to $N Y$; we have then an infinite number of infinite sectorial areas round $O$ which are all equivalent, though the angles are all unequal and ultimately tend to zero.

If the principles of hyperbolic geometry be assumed, let us consider the area of a sector of a circle $A^{\prime} A^{\prime}$ and of a strip $\mathrm{A}^{\prime} \mathrm{ABB}^{\prime}$ bounded by the straight lines $\mathrm{AB}, \mathrm{AA}^{\prime}, \mathrm{BB}^{\prime}$ and the arc of a curve $A^{\prime} B^{\prime}$ equidistant from the base AB. (Fig. 1.)

The area of the circular sector of radius $r$ and angle $\theta$ in "circular measure," i.e. the measure in which $2 \pi$ is the number of units in four right angles, is $2 \theta k^{2} \sinh ^{2} r / 2 k$, where $k$ is the space-constant. And the area of the strip bounded by the equidistant ourve is $k x \sinh r / k$, where $\mathrm{AB}=x$. The ratio of these is $\theta k / x \cdot \tanh r / 2 k$, which tends to a finite limit $\theta k / x$ as $r \rightarrow \infty$. Thus the sector and the strip appear to be infinities of the same order.

There still appears this paradox. If the area of the strip bears a finite ratio to the area of the sector, and the area of the sector bears a finite ratio to the area of the plane, the area of the strip should bear a finite ratio to the area of the plane; and yet it appears to require infinitely many identical strips laid side by side to cover the plane. The following considerations throw a different light upon this.

Consider any two strips whose bases are $\mathrm{AB}=x, \mathrm{CD}=y$, and let $y>x$. Place the strips with $\mathrm{CC}^{\prime}$ lying along $\mathrm{AA}^{\prime}$ and move the second strip to the right until $\mathrm{DD}^{\prime} \| \mathrm{BB}^{\prime}$. This is possible, since when $\mathrm{CD}$ lies on $\mathrm{AB}, \mathrm{DD}^{\prime}$ does not meet $\mathrm{BB}^{\prime}$, and if $\mathrm{CD}$ is moved sufficiently far to the right $\mathrm{DD}^{\prime}$ will cut $\mathrm{BB}^{\prime}$, as, for example, when 
$\mathrm{D}$ lies on $\mathrm{BB}^{\prime}$. Now draw a line $\mathrm{KK}^{\prime} \perp \mathrm{AA}^{\prime}$ and $\| \mathrm{BB}^{\prime}$; this line is also $\| \mathrm{DD}^{\prime}$. The figures $\mathrm{KABB}^{\prime}$ and $\mathrm{KCDD}^{\prime}$ each have the area $\frac{1}{2} k^{2} \pi$, and each strip therefore has the area $\frac{1}{2} k^{2} \pi+\mathbf{A}^{\prime} K^{\prime}$. Hence all strips have the same area.

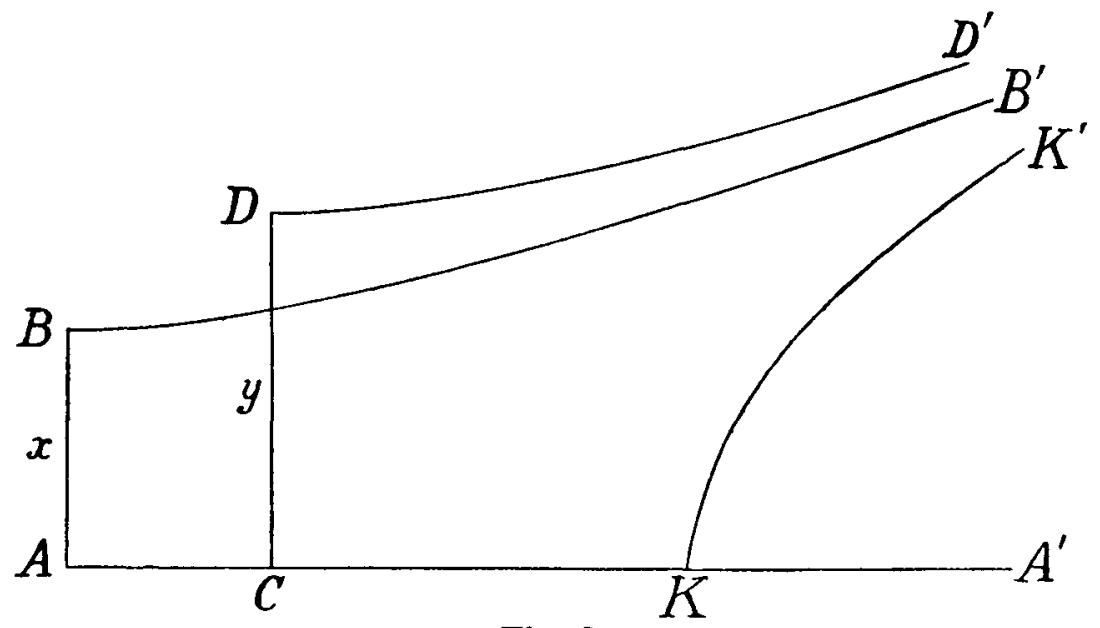

Fig. 2.

Again, the strip with the larger base can be placed so as to be included in the strip with the smaller base. It is only necessary to move CD until D lies on BB'. In fact, any strip may include an infinity of strips with the same base as its own. For KK' may be divided into an infinity of parts, each equal to $\mathrm{AB}$, and these form the bases of an infinity of strips, all of which are included within the strip $\mathrm{A}^{\prime} \mathrm{ABB}^{\prime}$. And each of these again can contain $\infty$ strips all identical with the first, and so on, so that any strip can contain $\infty$ strips identical with itself.

Such paradoxical results show that the notion of equality of infinite areas must be widely different from that of finite areas. Paradoxes of an entirely similar character are familiar in the theory of aggregates. Thus between the aggregate of all the rational numbers and that of the positive integers a $(1,1)$ correspondence can be established by arranging the rational numbers $p / q$ in rows such that in each row $p+q$ is constant. The number in each row is finite, and so each rational number has a definite numbered place. But, on the other hand, a $(\infty, 1)$ correspondence 
can also be established. For example, to the integer $n$ can be made to correspond the infinite aggregate of numbers $p / n$ where $p$ is prime to $n$. Two such aggregates are said to be equivalent or to have the same potency or cardinal number when there exists a $(1,1)$ correspondence between them. The area of an infinite strip is therefore analogous to the potency of an aggregate, and any two strips are equivalent since a $(1,1)$ correspondence can be established between pairs of equivalent elements.

These remarks have an interesting application to a proof of Euclid's axiom which was given by Legendre. ${ }^{*}$ Desiring to simplify Bertrand's proof by considering only infinite strips or, as he calls them, biangles, he proceeds as follows. AC, BD are two

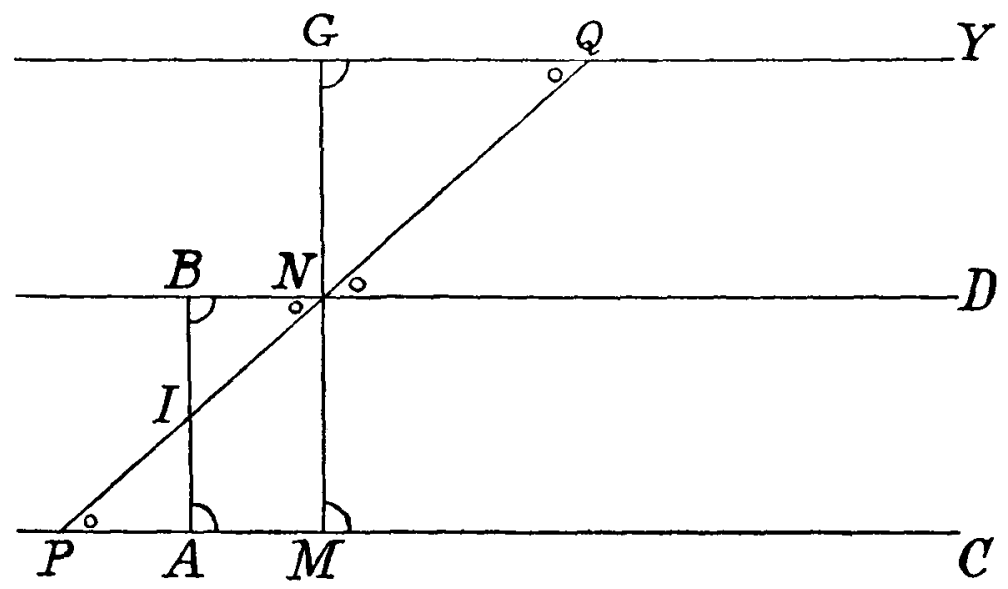

Fig. 3.

straight lines both perpendicular to $\mathrm{AB}$, and therefore forming a right biangle. At $M$, any point on $A C$, a perpendicular $M N$ is erected meeting $B D$ in $N$. It is required to prove (1) $M N=A B$, (2) $\mathrm{MN} \perp \mathrm{BD}$.

Join $N$ to $I$, the middle point of $A B$, and produce it to cut $A C$ in $P$, and also backwards to $Q$ so that $N Q=N P$, and through $Q$ draw GQY, making the angle GQN = QND. Also produce $M N$ to meet GY in $G$.

* A. M. Legendre, Réflexions sur différentes manières de démontrer la théorie des parallèles ou lo somme des trois angles du triangle. Paris, Mém. Acad. sc. Inst., xii. (1833), 367-410. \$ 18-23. 
Then it is easily proved that the angle $A P N=B N P=D N Q=N Q G$; the angle at $G$ is a right angle, and $N G=M N$. Then the right biangle CABD and the oblique biangle CPND are equivalent since the triangles API and BNI are congruent. So also the oblique biangles CPND and DNQY are superposable, and therefore equivalent, and the oblique biangle CPQY is equivalent to the right biangle CMGY. So far, everything is in agreement with the ideas of equivalence.

He proceeds. Hence CPQY, double of CPND, is equivalent to CMGY, and consequently double of the right biangle whose base is MN, half of MG. It follows from this that the oblique biangle CPND, which is equivalent to the right biangle whose base is $A B$, is equivalent also to the right biangle whose base is $M N$. But two equal right biangles must have their bases equal. Hence MN is equal to AB.

The second result, that $M N \perp B D$, follows easily from the result that $\mathrm{MN}=\mathrm{AB}$.

On reading over this proof for the first time, I was struck by what appeared to be a very crude argument in a circle. In referring to the right biangle whose base is $\mathrm{MN}$, Legendre appeared to assume that CMND is a right biangle, that is, that $M N \perp N D$, and this is only proved in the second part by the aid of the first part. Such a striking example of a circulus in probanclo by one of the foremost mathematicians appeared to me worthy of being recorded, and $I$ had prepared to bring it before the notice of the members of this Society. On going more closely into the matter, however, I discovered that the fallacy involved in Legendre's proof is of a much more subtle nature, and $I$ therefore cancelled my communication. Quite recently, however, I have come across a criticism of this proof by $\mathrm{H}$. Meikle, ${ }^{*}$ in which he makes the same mistake as I did, and I have therefore thought it worth while bringing up the matter again and vindicating Legendre from the charge of such an elementary fallacy.

The actual fallacy will be apparent in the light of the preceding remarks. We may admit that the right biangle with base $A B$ is equivalent to the right biangle with base $\mathrm{MN}$, as this in no way

* H. Meikle, On the theory of parallel lines. Edinburgh New Philas. J., xxxvi. (1844), p. 316 . 
implies that CMND is the biangle in question; but we cannot conclude that the bases $\mathrm{AB}$ and $\mathrm{MN}$ are therefore equal since we have seen that any two right biangles are equivalent, whatever be their bases.

It is only fair to add that Legendre perceived a difficulty in the proposition that two right biangles with equal bases are equal, for in ordinary geometry we have two such biangles CABD and CMND, with equal bases, but unequal since one contains the other. He explains this, however, in the usual way by remarking that their difference is infinitesimal compared with either of them. He did not appear to realise the difficulty in accepting the converse theorem that equivalent biangles have their bases equal. It is not necessary to go into non-Euclidean geometry to obtain equivalent biangles with unequal bases. If we take any two right biangles $\mathrm{A}^{\prime} \mathrm{ABB}$, $\mathrm{P}^{\prime} \mathrm{PQQ}$, a $(1,1)$. correspondence can be established between pairs of equivalent elements by dividing $\mathrm{AA}^{\prime}, \mathrm{PP}^{\prime}$ into parts inversely as $\mathrm{AB}, \mathrm{PQ}$.

Legendre's claim of having found at last " a demonstration of the theory of parallels, as simple as it is rigorous, and very suitable for being introduced into the elements," like all such claims before or since, breaks down, and the only course where rigour is aimed at is to state the assumptions clearly and frankly. 